\title{
Morphology as a risk factor for the malignant potential of $\mathbf{T} 2$ colorectal cancer
}

\author{
YUICHI MORI ${ }^{1}$, SHIN-EI KUDO $^{1}$, SHUNGO ENDO $^{2}$, CHIYO MAEDA $^{1}$, SHUMPEI MUKAI $^{1}$, \\ YASUHARU MAEDA ${ }^{1}$, SHINICHI KATAOKA ${ }^{1}$, KENICHI TAKEDA $^{1}$, KATSURO ICHIMASA $^{1}$, \\ HIDEYUKI MIYACHI $^{1}$, NARUHIKO SAWADA ${ }^{1}$, EIJI HIDAKA ${ }^{1}$ and FUMIO ISHIDA ${ }^{1}$ \\ ${ }^{1}$ Digestive Disease Center, Showa University Northern Yokohama Hospital, Yokohama 225-8503; \\ ${ }^{2}$ Department of Coloproctology, Aizu Medical Center, Fukushima Medical University, Aizuwakamatsu 965-0803, Japan
}

Received January 12, 2016; Accepted May 30, 2016

DOI: $10.3892 / \mathrm{mco} .2016 .951$

\begin{abstract}
Currently, depressed type T1 colorectal cancer is often detected and its malignant potential is being revealed. However, few reports have focused on the morphology of $\mathrm{T} 2$ colorectal cancer types. The aim of the present retrospective study was to clarify the characteristics of colorectal T2 cancer types derived from depressed type $\mathrm{T} 1$ cancer. The present study included 195 patients with colorectal T2 cancer surgically resected at a referral hospital between April 2001 and April 2009. A total of 48 lesions (25\%) with lymph node metastasis and $4(2 \%)$ with distant metastasis were found. The lesions were classified by their form during endoscopy into four categories: Group A, depressed type (the periphery consists of normal mucosa); group B, laterally spreading type; group C, protruded type; group $\mathrm{D}$, ulcerative type (the periphery consists of neoplastic mucosa). Tumor size, lymphatic and venous involvement, lymph node metastasis, and distant metastasis were compared between the four categories. The distribution of patients were as follows: Group A, 73 (37\%), group B, $26(13 \%)$, group C, 24 (12\%) and group D, $72(37 \%)$. The average tumor size for each category was $23,51,30$ and $36 \mathrm{~mm}$ for groups A, B, C and D, respectively. The number of positive findings for lymphatic involvement, venous involvement, lymph node metastasis and distant metastasis for each category were: Group A, 50 (69\%), 54 (74\%), 19 (26\%) and $1(1 \%)$; group B, $14(54 \%), 12(46 \%), 8(31 \%)$ and $0(0 \%)$; group C, $14(58 \%), 8(33 \%), 6(25 \%)$ and $0(0 \%)$; group D 35 (49\%), 41 (57\%), $16(22 \%)$ and 3 (4\%). Group A demonstrated a significantly smaller tumor size $(\mathrm{P}<0.01)$, and higher rates of lymphatic and venous involvement
\end{abstract}

\footnotetext{
Correspondence to: Dr Yuichi Mori, Digestive Disease Center, Showa University Northern Yokohama Hospital, 35-1 Chigasaki-Tyuo, Tsuzuki-Ku, Yokohama 225-8503, Japan

E-mail: ibusiginjp@hotmail.com
}

Key words: colorectal cancer, depressed type, gross appearance, macroscopic form, morphology
( $\mathrm{P}=0.0333$ and $\mathrm{P}=0.0019$, respectively). No significant differences were observed between categories with regards to nodal and distant metastases. Of the T2 cancer types, 40\% were the depressed type. Compared with the other forms, depressed type lesions exhibited a smaller tumor size, and higher rates of lymphatic and venous involvement, which suggested these lesions were of a more malignant nature.

\section{Introduction}

Numerous previous studies have investigated the clinicopathological characteristics of early colorectal cancer types. As a result, a consensus is forming in regards to the clinical treatment of T1 cancer types (1-3). According to the Colorectal Cancer Treatment guidelines, T1 colorectal cancer types are recommended for endoscopic treatment if they are papillary adenocarcinoma or tubular adenocarcinoma with a vertical invasion of $\leq 1,000 \mu \mathrm{m}$ and without any lymphovascular invasion (3).

T2 cancer types, which invade deeper layers as compared with $\mathrm{T} 1$ cancer types, are considered an early stage of advanced cancer. T2 cancer types are considered to be the developmental transition from early to advanced cancer. Although several retrospective studies on the association between morphology and tumor characteristics of $\mathrm{T} 2$ colorectal cancer types have been performed in Japan, there remains no consensus on the subject (4-8). This may be due to advanced cancer types exhibiting highly modified morphologies as compared with early cancer types.

Nevertheless, numerous lesions are thought to clearly derive from depressed type early cancer (Fig. 1). The present study proposed novel morphological categories for $\mathrm{T} 2$ colorectal cancer types, based on the growth and development route. The primary aim of the present study was to clarify the association between morphology and malignant potential of T2 colorectal cancer types.

\section{Patients and methods}

Patients. The present study included 195 patients (195 lesions) with T2 colorectal cancer who underwent surgical resection at Showa University Northern Yokohama Hospital (Yokohama, 
Japan) between April 2001 and April 2009. The present study retrospectively analyzed the association between morphology and clinicopathological factors. The present study was approved by the Ethics Committee of Showa University Northern Yokohama Hospital (Yokohama, Japan).

Groups and assessments. Preliminary categories were created for T2 cancer morphology, based on growth and development route. These were as follows: group A, depressed type; group B, laterally spreading type; group C, protruded type; group D, ulcerative type (Fig. 1). The morphologies containing depressed areas on the surfaces were divided into two groups (groups A and D). In other words, those lesions with some normal mucosa remaining in the margins of the depression were assigned to the group A, which was considered to be derived from depressed-type T1 cancer. Those with neoplastic mucosa around the entire circumference of the depression were assigned to the group D). Group B included laterally spreading flat component on its surrounding area, which was considered to be derived from laterally spreading type early $\mathrm{T} 1$ cancer. Group $\mathrm{C}$ included protruded type lesions that had purely protruded morphology without depression. The present study retrospectively investigated the association between these morphological classifications and tumor size, lymphovascular invasion, lymph node metastasis and distant metastasis.

Statistical analysis. The data were statistically analyzed using the $\chi^{2}$ test as a test of independence and the Mann-Whitney test for comparing the mean values of the two groups. $\mathrm{R}$ software (version 2.10.0; https://www.r-project.org/) was used for statistical analyses. In consideration of effects induced by multiple comparison, $\mathrm{P}<0.0125$ was considered to indicate a statistically significant difference by using Bonferroni's correction.

\section{Results}

Detailed description of the cases. Of the 195 patients, 48 exhibited lymph node metastasis and 4 were positive for distalmetastasis at the time of resection, accounting for 24.6 and $2.1 \%$ of the total number of cases, respectively. The number of cases in each classification included: Group A, 73 (37.4\%); group B, 26 (13.3\%); group C, 24 (12.3\%); group D, 72 (36.9\%) (Table I).

Association between T2 cancer morphology and tumor size.

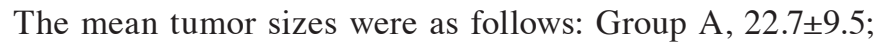
group B, 51.2 \pm 22.6 ; group C, 29.9 \pm 8.7 ; group D, 36.3 $\pm 11.3 \mathrm{~mm}$ (Table I). Depressed type T2 cancers were of a significantly smaller tumor size compared with the other types $(\mathrm{P}<0.01)$.

Association between T2 cancer morphology and vascular invasion. The positive rates for lymphatic invasion were as follows: Group A 68.5; group B, 53.8; group C 58.3; group D, 48.6\% (Table II). Depressed type T2 cancers exhibited higher positive rates for lymphatic invasion compared with the other types $(\mathrm{P}=0.0333)$.

The positive rates for venous invasion were as follows: Group A 74.0; group B 46.2; group C, 33.3; group D, 56.9\% (Table II). Depressed type T2 cancers had significantly higher positive rates for venous invasion compared with the other types $(\mathrm{P}=0.0019)$.
Table I. Number of patient lesions and tumor diameter of each morphological type.

\begin{tabular}{lcl}
\hline Morphology & No. patients (\%) & Tumor diameter \\
\hline Depressed type & $73(37.5)$ & $22.7 \pm 9.5 \mathrm{~mm}$ \\
Laterally spreading type & $26(13.3)$ & $51.2 \pm 22.6 \mathrm{~mm}$ \\
Protruded type & $24(12.3)$ & $29.9 \pm 8.7 \mathrm{~mm}$ \\
Ulcerative type & $72(36.9)$ & $36.3 \pm 11.3 \mathrm{~mm}$ \\
\hline
\end{tabular}

Table II. Lymphatic invasion and venous invasion of each morphological type.

\begin{tabular}{lrr}
\hline & \multicolumn{2}{c}{ No. patients $(\%)$} \\
\cline { 2 - 3 } Morphology & $\begin{array}{c}\text { Lymphatic } \\
\text { invasion }\end{array}$ & $\begin{array}{c}\text { Venous } \\
\text { invasion }\end{array}$ \\
\hline Depressed type $(\mathrm{n}=73)$ & $50(68.5)$ & $54(74.0)$ \\
Laterally spreading type $(\mathrm{n}=26)$ & $14(53.8)$ & $12(46.2)$ \\
Protruded type $(\mathrm{n}=24)$ & $14(58.3)$ & $8(33.3)$ \\
Ulcerative type $(\mathrm{n}=72)$ & $35(48.6)$ & $41(56.9)$ \\
\hline
\end{tabular}

Table III. Lymph node metastasis and distant metastasis of each morphological type.

\begin{tabular}{lcc}
\hline & \multicolumn{2}{c}{ No. patients $(\%)$} \\
\cline { 2 - 3 } Morphology & $\begin{array}{c}\text { Lymph node } \\
\text { metastasis }\end{array}$ & $\begin{array}{c}\text { Distant } \\
\text { metastasis }\end{array}$ \\
\hline Depressed type $(\mathrm{n}=73)$ & $19(26.0)$ & $1(1.4)$ \\
Laterally spreading type $(\mathrm{n}=26)$ & $8(30.8)$ & $0(0.0)$ \\
Protruded type $(\mathrm{n}=24)$ & $6(25.0)$ & $0(0.0)$ \\
Ulcerative type $(\mathrm{n}=72)$ & $16(22.2)$ & $3(4.2)$ \\
\hline
\end{tabular}

Association between T2 cancer morphology and lymph node metastasis/distant metastasis. The positive rates for lymph node metastasis were as follows: Group A, 26.0; group B, 30.8; group C, 25.0; group D, 22.2\% (Table III). No significant differences were observed between the four morphological classifications.

The positive rates for synchronous distant metastasis were as follows: Group A, 1.4; group B, 0; group C, 0; group D, 4.2\% (Table III). No significant differences were observed between the groups for distant metastasis.

\section{Discussion}

The present study proposed novel morphological classifications of T2 colorectal cancer with reference to growth and development route, and subsequently investigated their respective features.

Since numerous previous studies have investigated the growth and development route of $\mathrm{T} 1$ stage cancer, a consensus is forming on this issue $(1,2,9,10)$. Growth and development 


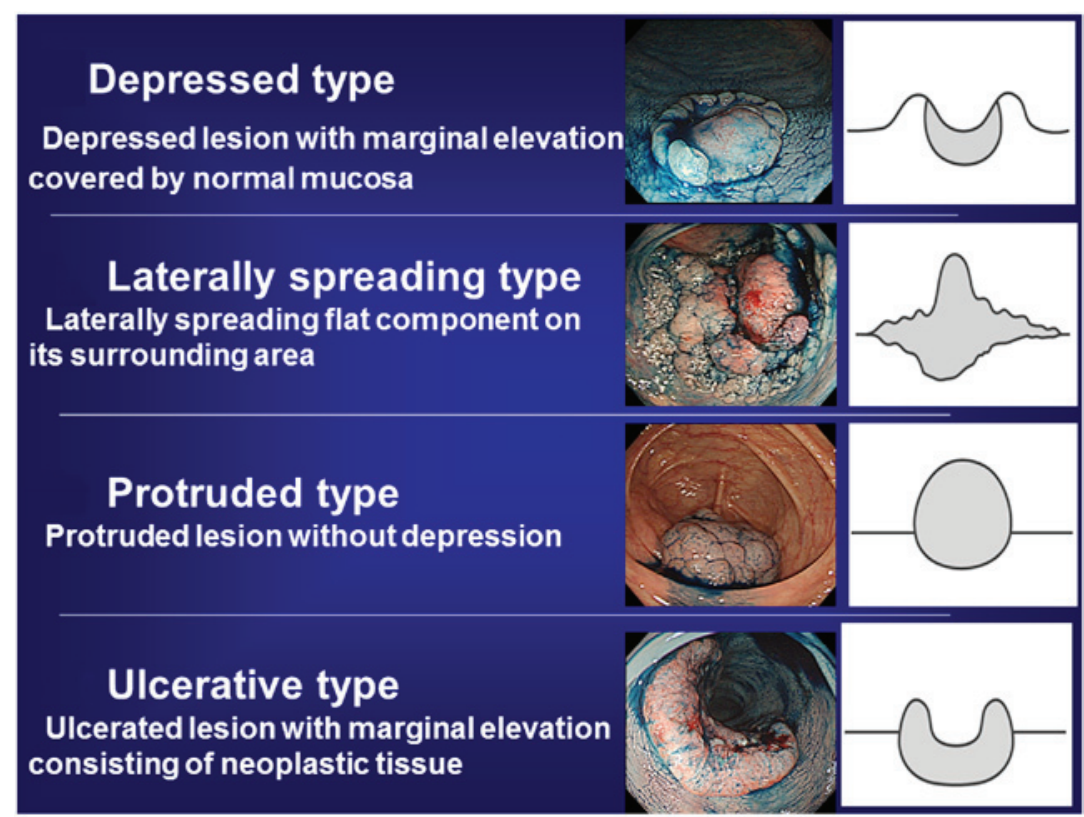

Figure 1. T2 cancer morphology classifications. The lesions were classified into four categories according to their form as viewed upon endoscopy.

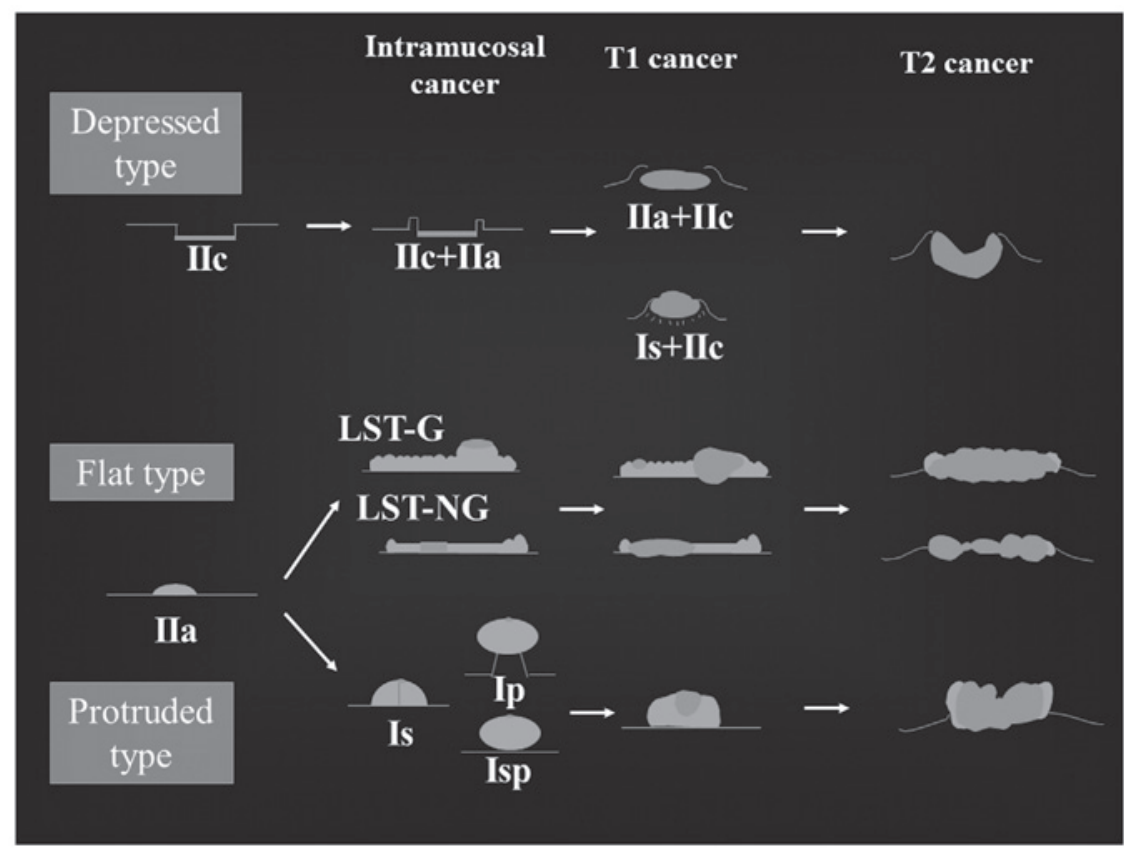

Figure 2. Assumed models of tumor growth during the development of colorectal neoplasia. Progression occurs in three distinct models.

classifications for depressed, flat and protruded types were constructed based on their various morphologies (Fig. 2). By acontrast, several previous studies on T2 cancer types have been previously performed (4-8); however, limited debate on this topic has occurred compared with that of T1 cancer types.

The morphologies containing depressed areas on tumor surfaces were dominant among T2-T4 cancer types, thus in the clinical setting they were conventionally treated as an identical morphology, often termed 'Bormann type 2' cancers in Japan. However, biological difference among the 'Bormann type 2' cancers were considered to exist, thus, several morphological classifications were proposed by Japan and the west. George et al (5) classified T2 cancers into polypoid and non-polypoid types based on whether the marginal mucosa is elevated or not. This resulted in $45 \%$ of tumors being classified as polypoid, half of which included adenomatous components within the lesions, and $48 \%$ were classified as non-polypoid, which rarely included adenomatous components. The remaining $7 \%$ included morphologies that exhibited both polypoid and non-polypoid characteristics. Non-polypoid tumor types often exhibited wild-type K-ras and had a significantly higher rate of postoperative recurrence compared with polypoid tumors, which suggested that non-polypoid tumors were more malignant. In a previous study of T2 colorectal cancer tumor types $\leq 2 \mathrm{~cm}$, Goi et al (6) reported that $63 \%$ of small, advanced colorectal cancer types were non-polypoid, 
while $\sim 25 \%$ of normal advanced tumors of $\geq 2 \mathrm{~cm}$ were non-polypoid tumors. The non-polypoid vs. polypoid classifications used by these researchers assumed that non-polypoid lesions were associated with the de novo cancer route (10) and that polypoid lesions were associated with the carcinogenic route that followed the adenoma-carcinoma sequence (9). Nevertheless, since these classification systems were focused on remnant intramucosal lesions, certain researchers may feel they should be restricted to use on early-stage cancer types. Another problem is that these non-polypoid vs. polypoid classifications were based on resected pathological specimens, thus, it cannot be used for treatment determination.

In consideration of these problems, the present study classified tumor types focusing on growth and development route based on preoperative endoscopic findings. Specifically, the laterally spreading and protruded types of lesions were firstly classified as groups B and C, respectively, because each were clearly derived from laterally spreading type and protruded type early lesions. The remaining depressed and ulcerative types of lesions were classified based on whether the margins have normal mucosal tissue or not. Depressed type lesions, which are thought to be de novo cancer-derived, were placed into their own category.

Of all the types in the present classification system, depressed type T2 cancer showed characteristic clinicopathological features. They exhibited a significantly smaller tumor size and higher incidence of lymphovascular invasion. These features have extremely strong resemblance to the clinicopathological features of depressed type $\mathrm{T} 1$ cancer $(1,2)$. However, no significant differences were observed in terms of lymph node metastasis and distant metastasis. This may be a result of the fact that positive rates for T2 cancer lymph node metastasis and distant metastasis were relatively low at 25.0 and $3.2 \%$, respectively. Therefore, this issue requires further study with a larger number of cases for analysis.
In conclusion, compared with the other types, depressed type T2 cancer exhibited a smaller tumor size, higher rate of lymphatic involvement and venous involvement, which suggested a more malignant nature of these lesions.

\section{References}

1. Kudo SE, Takemura O and Ohtsuka K: Flat and depressed types of early colorectal cancers: From East to West. Gastrointest Endosc Clin N Am 18: 581-593, 2008.

2. Kudo Se, Lambert R, Allen JI, Fujii H, Fujii T, Kashida H, Matsuda T, Mori M, Saito H, Shimoda T, et al: Nonpolypoid neoplastic lesions of the colorectal mucosa. Gastrointest Endosc 68 (4 Suppl): S3-S47, 2008.

3. Watanabe T, Itabashi M, Shimada Y, Tanaka S, Ito Y, Ajioka Y, Hamaguchi T, Hyodo I, Igarashi M, Ishida H, et al: Japanese society for cancer of the colon and rectum (JSCCR) guidelines 2014 for treatment of colorectal cancer. Int J Clin Oncol 20: 207-239, 2015.

4. Adachi, Yasuda, Kakisako, Sato, Shiraishi and Kitano: Histopathologic characteristics of advanced colorectal cancer smaller than $2 \mathrm{~cm}$ in size. Colorectal Dis 1: 19-22, 1999.

5. George SM, Mäkinen MJ, Jernvall P, Mäkelä J, Vihko P and Karttunen TJ: Classification of advanced colorectal carcinomas by tumor edge morphology: Evidence for different pathogenesis and significance of polypoid and nonpolypoid tumors. Cancer 89: 1901-1909, 2000.

6. Goi T, Kawasaki M, Hirono Y, Katayama K and Yamaguchi A: Clinicopathological analysis of invading muscularis propria (T2) cancers $<$ or $=20 \mathrm{~mm}$ in diameter. Int Surg 93: 1-5, 2008.

7. Miyamoto S, Boku N, Fujii T, Ohtsu A, Matsumoto S, Tajiri H, Yoshida S, Arai T, Ono M, Hasebe T and Ochiai A: Macroscopic typing with wall stricture sign may reflect tumor behaviors of advanced colorectal cancers. J Gastroenterol 36: $158-165,2001$

8. Zhang H, Chen CS, Cong JC, Qiao L, Hasegawa T and Takashima S: Clinicopathological characteristics of advanced colorectal cancer $30 \mathrm{~mm}$ or smaller in diameter. Chin Med Sci J 22: 98-103, 2007.

9. Morson BC: Precancerous and early malignant lesions of the large intestine. Br J Surg 55: 725-731, 1968.

10. Shimoda T, Ikegami M, Fujisaki J, Matsui T, Aizawa S and Ishikawa E: Early colorectal carcinoma with special reference to its development de novo. Cancer 64: 1138-1146, 1989. 